Case

Report

\title{
Ectopic Cervical Thymoma Excised through a Transcervical Approach Combined with Video-assisted Thoracoscopic Surgery: A Case Report
}

\author{
Shintaro Yokoyama, MD,${ }^{1}$ Ryozo Hayashida, MD, ${ }^{2}$ Koichi Yoshiyama, MD, ${ }^{1}$ \\ Kunihiro Ozaki, MD, ${ }^{2}$ Toshihiro Matsuo, MD ${ }^{2}$ Shinzo Takamori, MD, ${ }^{1}$ \\ and Yoshito Akagi, $\mathrm{MD}^{1}$
}

\begin{abstract}
Ectopic cervical thymoma (ECT) is a rare tumor. We present a case of 56-year-old woman with an ECT in the anterior neck that was correctly diagnosed preoperatively. The patient had no symptoms of myasthenia gravis or other immune disorders, and the tumor was not invading any adjacent structures. We performed tumor resection and thymectomy through a transcervical approach using video-assisted thoracoscopic surgery with a multi-access single port. To our knowledge, this is a novel combined technique for the resection of an ECT.
\end{abstract}

Keywords: ectopic cervical thymoma, thymectomy, transcervical approach, video-assisted thoracoscopic surgery.

\section{Introduction}

Only a few cases of ectopic cervical thymoma (ECT) have been reported. Most ECTs occur along the migration route of the thymus during the fetal period and are thought to arise from ectopic thymic tissue. ECT might be misdiagnosed as a thyroid tumor or lymphoma because of its location and prevalence. Specific therapeutic approaches are needed in ECT, thyroid tumor, and lymphoma. Therefore a correct diagnosis is essential. Here, we report a case of an ECT that was correctly diagnosed before surgery and was resected through a transcervical approach

${ }^{1}$ Department of Surgery, Kurume University School of Medicine, Kurume, Fukuoka, Japan

${ }^{2}$ Department of Surgery, Oita Prefecture Saiseikai Hita Hospital, Hita, Oita, Japan

Received: October 14, 2014; Accepted: December 8, 2014 Corresponding author: Shintaro Yokoyama, MD. Department of Surgery, Kurume University School of Medicine, 67 Asahi-machi, Kurume, Fukuoka 830-0011, Japan

Email: yokoyama_shintarou@med.kurume-u.ac.jp

(C)2015 The Editorial Committee of Annals of Thoracic and Cardiovascular Surgery. All rights reserved. combined with video-assisted thoracoscopic surgery for thymectomy.

\section{Case Report}

A previously healthy 56-year-old woman visited our hospital for worsening dyspnea and neck swelling. She had first noticed the neck swelling about 5 months prior but disregarded it because of no other symptoms. On physical examination, the center of her neck was swollen above the suprasternal notch, and an immovable elastic hard mass was palpable. On auscultation, we heard tracheal stenotic sounds near the mass. She had no symptoms of myasthenia gravis. A hematological examination indicated normal findings, including normal tumor marker levels of carcinoembryonic antigen, squamous cell carcinomarelated antigen, alpha-fetoprotein, soluble interleukin-2 receptor, and thyroglobulin. The titer of anti-acetylcholine receptor antibodies was not elevated. A chest radiograph revealed a round tumor at the upper mediastinum. Computed tomography showed a quadrangular tumor (dimensions, $65 \mathrm{~mm} \times 63 \mathrm{~mm} \times 45 \mathrm{~mm}$ ) in front of the trachea (Fig. 1a, b). Magnetic resonance imaging showed a 


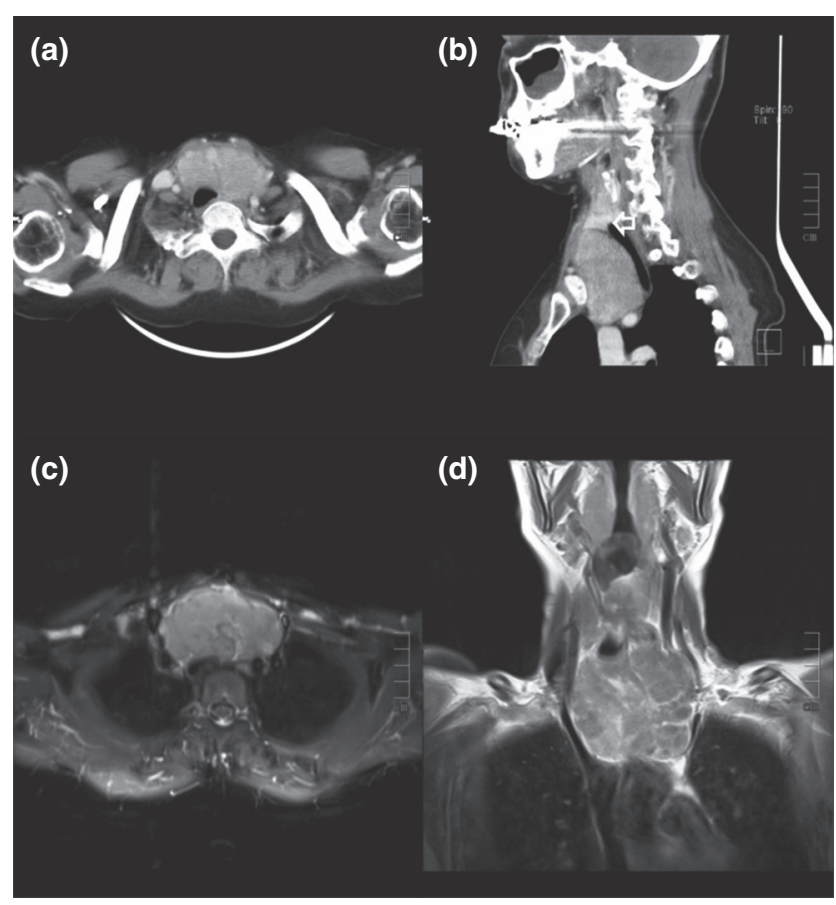

Fig. 1 Computed tomography (CT) and magnetic resonance image (MRI). (a, b) Contrast enhanced CT showing a lesion oppressing the trachea and thyroid (arrow). (c, d) MRI showing a well-defined round lesion with multilocular structures. Gadolinium contrast enhancing MRI revealing the lesion with slow enhancement.

well-defined tumor that did not invade the trachea, carotid artery, jugular vein, or any other neighboring structure. The tumor presented with multiform gadolinium slow enhancement (Fig. 1c, d) with multiple segmentations separated by a fibrous septum. A percutaneous fine needlepunched biopsy showed lymphocytes spread, with some atypical polygonal cells positive for AE1/AE3 on immunohistochemical analysis. Accordingly, the tumor was diagnosed as an ectopic type B1 thymoma in the cervical region.

ECT resection and thymectomy were performed. First, we excised the ECT and the left upper pole of the thymus through a collar incision (Fig. 2a). The tumor was dissected easily from the adjacent structures, including the trachea and bilateral carotid arteries and jugular veins without invasion. Grossly, the ECT was connected to the left upper pole of the thymus. We dissected the thymus caudally and incised it at the point it merged at the right lobe of the thymus and then removed the ECT without disconnecting it from the left upper pole. Subsequently, the right superior pole was similarly dissected caudally from the collar incision, to point at which it met the left lobe. In the series of dissections around the upper pole, the thymic veins were each ligated and divided. We then inserted a multi-access single port from the subxiphoid space toward the anterior mediastinum (Fig. 2b). The video-assisted thoracoscopic surgery through this port in the cranial direction easily allowed for complete dissection of both lower poles of the thymus without any remaining thymic tissues.

The histopathological findings showed both lymphocytepoor spindle cells and lymphocyte-rich polygonal cells. Based on these findings, the tumor was confirmed as a type AB thymoma according to the 2004 World Health Organization (WHO) histological classification. The tumor cells had microscopic invasion to fat tissues around the capsule membrane of the tumor that was equivalent to Masaoka stage II. However, it should be noted that no microscopic connection was seen between the thymoma and the thymic tissue.

The postoperative course was uneventful. At 1 year and 3 months after surgery, the patient is doing well with no evidence of recurrence.

\section{Discussion}

During the embryonic period, the thymus develops from the third and fourth pharyngeal pouches by the fifth week of gestation, with cooperation between epithelial cells of the pharyngeal pouch and mesenchymal cells derived from the neural crest. ${ }^{1)}$ The thymus anlage then descends caudally into the anterior mediastinum at around the eighth week of gestation. Ectopic thymoma may develop from the remnant thymic tissues anywhere along this descending route. Most prior reports of ectopic thymoma have indicated that the tumor was located near the thyroid via the normal location of the thymus in the anterior mediastinum, while a few rare reports have indicated that the ectopic thymoma was originated in the thyroid, lung, or right pleura. ${ }^{2-4)}$ To date, there have been no reports of ectopic thymomas in other locations. However, some cases of ectopic thymic cyst occurring in the posterior mediastinum have been reported.5) Those findings suggested that ectopic thymoma may arise from the posterior mediastinum.

A report by Chan and Rosai analyzed the morphology and clinical behavior of neck tumors with thymic or related branchial pouch differentiation. ${ }^{6}$ ) These authors classified these tumors into four subtypes: hamartomatous thymoma, ECT, spindle epithelial tumor with thymus-like differentiation, and carcinoma showing thymus-like differentiation. Among these, ECTs were described as "histologically 


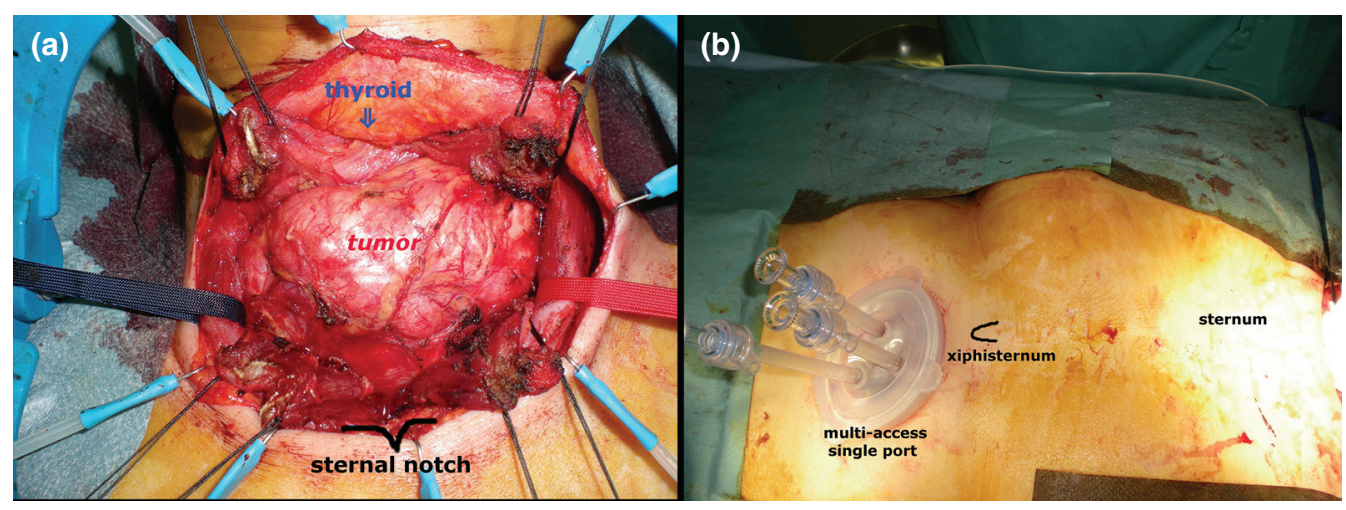

Fig. 2 The operative view. (a) Neck tumor dissected through a collar incision indicating the adjacent structures near the tumor. Surgically, no invasion into the thyroid was seen. (b) A multi-access single port inserted from the subxiphoid space toward the anterior mediastinum.

Table 1 Clinicopathological features of ECT in previous 26 studies

\begin{tabular}{|c|c|c|}
\hline Parameter & No. & $\%$ \\
\hline Total & 26 & \\
\hline \multicolumn{3}{|l|}{ Sex } \\
\hline Male & 6 & $23.1 \%$ \\
\hline Female & 17 & $65.4 \%$ \\
\hline Unknown & 3 & $11.5 \%$ \\
\hline \multicolumn{3}{|l|}{ Age (years) } \\
\hline Median (range) & $53.5(32-86)$ & \\
\hline$\leq 50$ & 8 & $30.8 \%$ \\
\hline $51-70$ & 12 & $46.2 \%$ \\
\hline$\geq 71$ & 4 & $15.4 \%$ \\
\hline Unknown & 2 & $7.7 \%$ \\
\hline \multicolumn{3}{|l|}{ Location } \\
\hline Left neck & 13 & $50.0 \%$ \\
\hline Right neck & 8 & $30.8 \%$ \\
\hline Center of the neck & 3 & $11.5 \%$ \\
\hline Unknown & 2 & $7.7 \%$ \\
\hline \multicolumn{3}{|l|}{ WHO histological classification } \\
\hline Type A & 4 & $15.4 \%$ \\
\hline Type AB & 9 & $34.6 \%$ \\
\hline Type B1 & 3 & $11.5 \%$ \\
\hline Type B2 & 2 & $7.7 \%$ \\
\hline Type B3 & 1 & $3.8 \%$ \\
\hline 'Invasive thymoma' & 3 & $11.5 \%$ \\
\hline 'Thymoma' & 4 & $15.4 \%$ \\
\hline \multicolumn{3}{|l|}{ Immunological disorders* } \\
\hline Myasthenia gravis & 9 & $34.6 \%$ \\
\hline Hypogloburinemia (Good's syndrome) & 1 & $3.8 \%$ \\
\hline ITP & 1 & $3.8 \%$ \\
\hline Lichen planus & 1 & $3.8 \%$ \\
\hline None & 16 & $61.5 \%$ \\
\hline \multicolumn{3}{|l|}{ Malignant behavior } \\
\hline Invasion or recurrence** & 5 & $19.2 \%$ \\
\hline Absent & 21 & $80.8 \%$ \\
\hline
\end{tabular}


identical to mediastinal thymomas, and residual ectopic thymus is not uncommonly identifiable in the periphery of the tumor." ${ }^{\text {(6) }}$ Our case also had no residual ectopic thymic tissues, as observed on histological analysis. Hence, the histological connection between a thymoma and remnant thymic tissues does not seem to define whether it is ECT or not.

To the best of our knowledge, 74 cases of a neck tumor with thymic or related branchial pouch differentiation have been reported in the English literature. Among these, only 26 cases had ECT. Table 1 shows the clinicopathological characteristics of these ECT cases. These tumors are more common in women $(65.4 \% ; 17$ cases $)$ with a median age of 53.5 years (range, 32-86 years). The ECTs were on the left side of the neck in 13 cases (50.0\%), on the right side in eight cases $(30.8 \%)$, at the center above the sternum in three cases $(11.5 \%)$, and unknown in two cases $(7.7 \%)$. Half of the ECTs cases were located in the left side of the neck. One reason for this lateral predominance may be delayed migration of the upper pole of the thymic left lobe that is derived from the fourth pharyngeal pouch. ${ }^{7)}$ According to the WHO histological classification, the ECT was type A in four cases (15.4\%), type $\mathrm{AB}$ in nine cases (34.6\%), type B1 in three cases (11.5\%), type B2 in two cases (7.7\%), type B3 in one case $(3.8 \%)$, "invasive thymoma" in three cases $(11.5 \%)$, and "thymoma" in four cases $(15.4 \%)$. Moreover, nine cases $(34.6 \%)$ were complicated by myasthenia gravis. This proportion seems slightly lower than that of patients with mediastinal thymoma. ${ }^{8}$ In addition, extremely rare cases have been complicated by idiopathic thrombocytopenic purpura, lichen planus, and Good's syndrome. ${ }^{9}{ }^{10)}$ Malignant behavior, such as direct invasion or recurrence, was reported in three cases $(11.5 \%)$. Although the number of cases is small, no cases of ECT showing direct infiltration of the adjacent vessels such as the carotid artery and jugular vein have been reported. This suggests that there is a high probability that complete ECT resection can be achieved.

ECT may be misdiagnosed as thyroid cancer or lymphoma because of its location and difficulty evaluating its histological features. In fact, a few authors have reported cases misdiagnosed based on histological findings from preoperative biopsy. ${ }^{10)}$ Most of those cases were difficult to distinguish from thyroid carcinoma or T-cell lymphoblastic lymphoma. We performed percutaneous fine needle-punched biopsy using a size 18 gauge needle under ultrasonography and found a type B thymoma component. However, we should keep in mind that the two cell patterns, commonly seen in type B thymoma, consisting of thymic epithelial cells and small immature lymphocytes, are not always found, as the biopsy tissue extracted is not sufficient to make such as diagnosis.

We performed thymectomy after ECT resection. We selected the transcervical approach combined with videoassisted thoracoscopic surgery using a multi-access single port. The ECT was excised in wide operation field secured by a collar incision, and the bilateral upper poles of the thymus were also resected directly. Consecutively, video-assisted thoracoscopic thymectomy through a multi-access single port was performed.

Transcervical thymectomy was first proposed by Crile in 1966, ${ }^{11)}$ and Bril and Cooper further developed the technique. ${ }^{12)}$ They placed the patient in a hyperextended neck position, used an original retractor under the sternum, and performed the thymectomy through a small collar incision. This procedure has been widely adopted for thymo-thymectomy. We performed the operation in our case using their approach as a reference. To the best of our knowledge, our report is the first description of our efficient combined approach for ECT resection and thymectomy. Since both upper poles of the thymus had already been resected, the residual thymus could be easily and safely excised using the thoracoscopic procedure. Thus, our approach was confirmed to be an effective method for treating ECT.

\section{Conclusion}

When encountering a neck tumor, clinician should be aware of the rare ECT. Our combined technique for ECT resection and thymectomy through a trans-cervical approach using video-assisted thoracoscopic surgery with a multi-access single port was effective, is an option for surgical resection, and is recommended for ECT treatment.

\section{Disclosure Statement}

The authors have no financial or other conflicts of interest to declare.

\section{References}

1) Varga I, Pospisilova V, Jablonska-Mestanova $V$, et al. The thymus: picture review of human thymus prenatal development. Bratisl Lek Listy 2011; 112: 368-76.

2) Cohen JB, Troxell M, Kong CS, et al. Ectopic intrathyroidal thymoma: a case report and review. Thyroid 2003; 13: 305-8. 
3) Skoutelis K, Nikolopoulos DD, Markopoulos K, et al. Ectopic thymoma of the lung; a rare case report and review of the literature. Cases J 2009; 2: 9149.

4) Kitada M, Sato K, Matsuda Y, et al. Ectopic thymoma presenting as a giant intrathoracic tumor: a case report. World J Surg Oncol 2011; 9: 66.

5) Ihaya A, Chiba $Y$, Inoue $H$, et al. An operated case of ectopic thymic cyst of the posterior mediastinum. Kyoubu Geka 1990; 43: 317-20. (in Japanese)

6) Chan JK, Rosai J. Tumors of the neck showing thymic or related branchial pouch differentiation: a unifying concept. Hum Pathol 1991; 22: 349-67.

7) Matsutani Y. Embryological study on aberrant thymus (author's transl) Nihon Kyobu Geka Gakkai Zasshi 1980; 28: 1403-12. (in Japanese)

8) Okumura M, Ohta M, Tateyama H, et al. The World Health Organization histologic classification system reflects the oncologic behavior of thymoma: a clinical study of 273 patients. Cancer 2002; 94: 624-32.

9) Mineo TC, Biancari F, D’Andrea V. Myasthenia gravis, psychiatric disturbances, idiopathic thrombocytopenic purpura, and lichen planus associated with cervical thymoma. J Thorac Cardiovasc Surg 1996; 111: 486-7.

10) Nagoya A, Kanzaki R, Nakagiri T, et al. Ectopic cervical thymoma accompanied by Good's syndrome. Annals of Thoracic and Cardiovascular Surgery 2013; 12: 02027.

11) Crile G. Thymectomy through the neck. Surgery 1966; 59: 213-5.

12) Bril V, Kojic J, Ilse WK, et al. Long-term clinical outcome after transcervical thymectomy for myasthenia gravis. Annals of Thoracic Surgery 1988; 65: $1520-2$. 